\title{
The genetic consequences of habitat fragmentation: the case of forests
}

\author{
Piotti A \\ Habitat fragmentation is one of the most serious threats to plant biodiversity at \\ the within-population level. Growing attention on the genetic effects of habitat \\ fragmentation is reflected in the 2008 publication of several review papers. In \\ general, fragmentation showed a negative effect on the genetic variability of \\ plant populations. However, for forest trees the genetic signal of fragmenta- \\ tion seems less clear. Here I discuss the development of less explored issues \\ that can help to clarify some unresolved questions about tree responses to \\ fragmentation. In particular, the understudied effect of delay in sexual matur- \\ ity and the need for accurate estimates of gene flow are taken into account. I \\ finally underline the potential role of the Italian peninsula as an open-sky \\ laboratory for forest fragmentation studies.
}

Keywords: Conservation genetics, Range limits, Genetic diversity, Gene flow

Much attention has been paid in recent years to the effect of habitat fragmentation on plant population genetics. Searching the ISI Web of Knowledge database using a combination of "fragmentation" and "genetic diversity" (or "gene flow") and "plant*" (or "tree*") I obtained 442 publications since 1992 (cited 7388 times), more than a half published after 2005. In 2008 some enlightening review papers were published, reporting apparently controversial results on the relationship between fragmentation and genetic diversity (Aguilar et al. 2008, Eckert et al. 2008, Kramer et al. 2008).

Genetic consequences of fragmentation are suggested by the classical theory of population genetics. At the population level, the fragmentation process reduces population size and increases isolation determining genetic bottlenecks. Remnant populations experience increased genetic drift, elevated inbreeding and limited gene flow from surrounding populations. This is expected to lead to decreasing genetic variability and increasing differentiation among remnant populations. The smaller the population size the greater these effects on genetic structure (Young et al. 1996).

Department of Environmental Sciences, University of Parma, v.le Usberti 11/A, I43100 Parma (Italy)

\section{@, Andrea Piotti (andre@dsa.unipr.it)}

Received: Jan 12, 2009 - Accepted: Mar 31, 2009

Citation: Piotti A, 2009. The genetic consequences of habitat fragmentation: the case of forests. iForest 2: 75-76 [online: 2009-06-10] URL:

http://www.sisef.it/iforest/show.php? id $=496$
In a recent paper, Aguilar et al. (2008) reviewed 101 studies on the effects of fragmentation on plant species and found a significant reduction in genetic variability in remnant populations. They draw the conclusion that habitat fragmentation decreases the genetic diversity of plant populations. This trend, more pronounced for common and outcrossing species, seems strongly dependent on the time elapsed from fragmentation: the older the fragmentation event the higher the loss of genetic variability.

A previous review on the "central-marginal hypothesis" by Eckert et al. (2008) also found reduced variability in fragmented populations: in $68 \%$ of the considered studies peripheral populations (typically smaller and more fragmented than central ones) experienced a decline in genetic diversity. Ohsawa \& Ide (2008) review of the distribution of genetic variability along altitudinal transects confirms that a reduction of genetic variability in peripheral plant populations is quite common. In half of the studies high altitude populations, usually more scattered, are characterized by lower genetic variability than medium altitude ones (whereas the opposite result was found in only $20 \%$ of cases).

Apparently quite different results were found by Kramer et al. (2008). Reviewing some studies on genetic structure and levels of gene flow in fragmented tree populations they hypothesized that forest trees might be an exception to theoretical expectations. They assert that empirical support for expected effects is scarce and as a consequence they proposed that a "paradox of forest fragmentation genetics" exists. They primarily ascribe this lack of evidence to extensive gene flow characteristic of forest trees and to a methodological flaw: the fragmentation cases studied would be of too recent origin to have produced recognizable genetic signals. As a consequence, they put forward some suggestions: 1) since forest species do not show similar responses to fragmentation, future studies should carefully take into account species-specific ecological realities; 2) ecological consequences of fragmentation, such as reduced pollen and seed production or recruitment failure, can frequently be more urgent to assess than reduction of genetic variability.

So why this discrepancy? A closer look at the results shows that Kramer et al. (2008) did not claim to have carried out a rigorous literature review. Their conclusions are intentionally and intelligently challenging. On the other hand, less than $20 \%$ of the plant species analyzed by Aguilar et al. (2008) are trees, even though the vast majority of reviewed studies were on long lived species (perennial herbs). Results by Eckert et al. (2008) were obtained analyzing studies on both plants and animals, when they considered only conifers the percentage of studies showing a reduction in genetic variability decreased from $68 \%$ to $54 \%$. Therefore the truth lies somewhere in the middle. In fact, what it is really persistent is this fifty-fifty trend that suggests that trees might not behave like herbaceous plant and animal: their responses seem somehow more variable.

Trees can certainly buffer, or at least delay, the effects of fragmentation because of typically high gene flow and long life cycles, as discussed by Kramer et al. (2008). But given the high variability of forest tree responses to habitat fragmentation these two key-factors deserve a deeper analysis, focusing on what differentiate trees from other plants.

In addition to a very long life span many tree species are characterized by a marked delay in sexual maturity that can determine a peculiar behavior in ecological processes. For instance, due to an extended juvenile phase, in recently colonized areas, established individuals do not contribute for decades to regeneration. Hence gene flow from outside represents the only source of new colonizers. Austerlitz et al. (2000) showed that this can markedly reduce the effect of genetic bottlenecks in subsequent colonization events during tree migrations, explaining the low among-population differentiation that usually characterized forest trees. Delay in sexual maturity can therefore further increase the "genetic resilience" to fragmentation of some tree species, with respect to what Kramer et al. (2008) hypothesized.

But probably gene flow plays the lead in this intricate plot. The study of gene flow or, with a brand new definition, of the 'movement ecology' of a species in a fragmentation context is crucial to understand how really isolated is a geographically isolated 
population (Aguilar et al. 2008, Nathan 2008). It is known that forest trees show extensive gene flow, especially when the wind is the dispersal vector for pollen or seeds. If a remnant population, hundreds of kilometers apart from the nearest one, has an immigration rate sufficiently high to counterbalance genetic drift, it can maintain its genetic variability. Until now gene flow in fragmented population has been studied by means of indirect genetic methods (i.e., $\mathrm{F}_{\mathrm{ST}}$-based methods). The results obtained with this approach do not tell us what is the current gene flow pattern, they only summarize what the history of a population has determined (e.g., how much gene flow has occurred over the last $n$ generations). On the contrary, direct methods (i.e. parentage analysis) furnish accurate estimate of contemporary gene flow (e.g. how many foreign seeds have immigrated and established, say, during the last 10 years). Such estimates allow to detect instantaneous signals produced by fragmentation. There is a "clear gap in the literature of plant population genetics that precluded us making further generalization" stated Aguilar et al. (2008) particularly referring to parentage studies. The assessment of gene flow in fragmented population at distribution edges, for example, will be crucial to solve one among the most intriguing ecological topics: why does adaptation fail at range margins? As Brindle \& Vines (2006) pointed out, two contrasting hypotheses can be advanced. If fragmented peripheral populations experimented high gene flow rate, alleles with adaptive value could be lost because of immigration of locally deleterious alleles from central populations. If gene flow is restricted adaptation can on the contrary be hampered by the rapid decrease of genetic variability because Allee effect and genetic drift prevail. In such scenario it will be important to explore whether asymmetries in emigration and immigration rates between large (usually central) and small (peripheral) populations can influence the adaptive potential in fragmented populations.

Experiments designed for the comparison among increasing levels of fragmentation can help to understand how and on what geographical scale gene flow patterns vary, and, eventually, to highlight ecologically relevant thresholds a fragmented population should not go over. Accurate estimates of gene flow in fragmented populations might give a boost to the comprehension of current and future responses of forest trees to fragmentation and certainly will help to design species-specific conservation strategies. The Italian peninsula served as glacial refugium where much genetic variability has been conserved during past glaciations, it represents an important European hot spot for biodiversity and nowadays is the rear edge of the distribution of many forest tree species. Italy represents an open-sky laboratory for forest fragmentation studies. Nevertheless, little efforts have been done up to now for testing the hypotheses suggested by theoretical and experimental evidence.

\section{References}

Aguilar R, Quesada M, Ashworth L, HerreriasDiego Y, Lobo J (2008). Genetic consequences of habitat fragmentation in plant populations: susceptible signals in plant traits and methodological approaches. Molecular Ecology 17: 51775188. - doi: 10.1111/j.1365-294X.2008.03971.x Austerlitz F, Mariette S, Machon N, Gouyon PH, Godelle B (2000). Effects of colonization processes on genetic diversity: differences between annual plants and tree species. Genetics 154: 1309-1321. [online] URL: http://www.genetics.org/cgi/content/abstract/154/3/1309

Brindle JR, Vines TH (2006). Limit to evolution at range margins: when and why does adaptation fail? Trends in Ecology and Evolution 22: 140147. - doi: 10.1016/j.tree.2006.11.002

Eckert CG, Samis KE, Lougheed SC (2008). Genetic variation across species' geographical ranges: the central-marginal hypothesis and beyond. Molecular Ecology 17: 1170-1188. - doi: 10.1111/j.1365-294X.2007.03659.x

Kramer AT, Ison JL, Ashley MV, Howe HF (2008). The paradox of forest fragmentation genetics. Conservation Biology 22: 878-885. - doi: 10.1111/j.1523-1739.2008.00944.x

Nathan R (2008). An emerging movement ecology paradigm. Proceedings of the National Society of Science 105:19050-19051. - doi: 10.1073/pnas. 0808918105

Ohsawa T, Ide Y (2008). Global patterns of genetic variation in plant species along vertical and horizontal gradients on mountains. Global Ecology and Biogeography 17: 152-163. - doi: 10.1111/j.1466-8238.2007.00357.x

Young A, Boyle T, Brown T (1996). The population genetic consequences of habitat fragmentation for plants. Trends in Ecology and Evolution 11: 413-418. - doi: 10.1016/0169-5347(96) 10045-8 